\title{
Simulate Separation Influence on Company
}

\author{
Fan Yang \\ North China Electric Power University, \\ Baoding,Hebei,China \\ 406624985@qq.com.
}

\begin{abstract}
Recent years, more and more company have focused on their human resources management, and separation becomes a very important factor. This thesis analyzes the influence of separation rate on company, so as to manage company better. First, we apply Analytic Network Process to construct a dynamic model, studying the factors which affect the separation rate. Second, thesis explains the way of separation infection and assess direct and indirect effect of separation on productivity. Last, we use Matlab to simulate the situation when company cannot control separation, it will appear the "separation explosion", then manager can early avoid to fluctuations in employees system. Through simulation, company will develop healthier and healthier in long-term. In results, employee's level and department have a tightly relationship with separation, and this stir can o diffuse from employee to employee. This report supplies method to identify those people that are likely to leave to prevent further churning. The multi-layer complex networks we get are convenient to evaluate the operational status at multi-angle. Company can use it to improve the management. On this condition, the company will develop healthier in long-term.
\end{abstract}

Keywords-Analytic Network Process; productivity; departure infection; fluctuations; simulation

\section{INTRODUCTION}

What' $s$ the secret of company' $s$ success? In the final word, is the talented person. Good human resources management is essential for a company. But nowadays, with the increasing fierce of competition, it becomes more and more difficult to find the talent. HR not only need to recruit the best person, but also need to keep those high-quality employees[i]. To help company reach this goal better, we use human resources network to simulate possible employees' movement. Through analyzing the model, users can know the company how to develop clearly, then forecast its development tend. Meanwhile, HR can find the change laws of personnel movement[ii], so as to prepare well in advance and increase the productivity. Finally, the company will obtain greater benefits.

\section{MAIN CAUSE OF SEPARATION RATE}

In the human resources network, separation rate is an important factor affecting the organization balance. When a person changes occurred in the position in the network, can stir network ${ }^{[i i i]}$ that was relatively stable, and drive fluctuations of the surrounding nodes. Here, we first analyze the factors lead to separation.

Term: Normally,the more year a person on duty, the less he/she will quit.

Ability: Something shows personal work completion.

Department: Different sectors have different churn rate.

Level: Churn rate is influenced by the worker's level.

Loyalty: The less the loyalty, the higher the churn rate.

Other factor $\varepsilon$ : Some factors that cannot be accurately identified but have a certain

influence.

We believe that the separation rate is related to six factors: term, ability, department, level, loyalty and other factor $\varepsilon$

We use the Analytic Network Process, which referred to as ANP, to analyze and sort the factor of churn rate. The analytic network process(ANP) is a structured technique for organizing and analyzing complex decisions, based on mathematics and psychology. What's more, in many practice problems, there are not only control but also feedback between high-layers and low-layers. And each element is not completely independent, it has relationship to other elements. So we use ANP instead of AHP(Analytic Hierarchy Process). It was developed by Satty in the 1996 and has been extensively studied and refined since then.

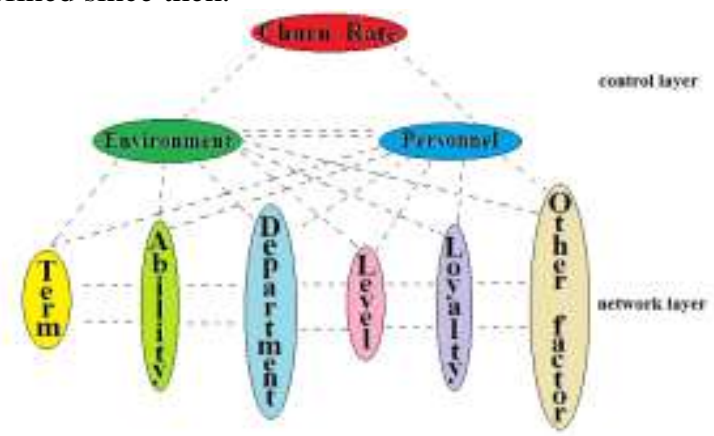

Figure 1. ANP model

In order to discuss the weight percentage among the variables, we want to establish the correlation matrix for each indicator ${ }^{[i v]}$. 
TABLE I . WEIGHT ALLOCATION

\begin{tabular}{|r|l|l|l|l|l|l|}
\hline & Term & Ability & $\begin{array}{l}\text { Depart } \\
\text { ment }\end{array}$ & Level & $\begin{array}{l}\text { Loyal } \\
\text { ty }\end{array}$ & $\varepsilon$ \\
\hline Term & 1 & $1 / 5$ & $1 / 3$ & $1 / 3$ & 3 & $1 / 2$ \\
\hline Ability & 5 & 1 & 3 & 3 & 2 & 2 \\
\hline $\begin{array}{r}\text { Depart } \\
\text { ment }\end{array}$ & 3 & $1 / 3$ & 1 & $1 / 2$ & 3 & 1 \\
\hline Level & 3 & $1 / 3$ & 2 & 1 & 2 & 1 \\
\hline Loyalty & $1 / 3$ & $1 / 2$ & 1 & 1 & 1 & 2 \\
\hline$\varepsilon$ & 2 & $1 / 2$ & 1 & 1 & $1 / 2$ & 1 \\
\hline
\end{tabular}

We use $\omega_{i j}$ to reflect the importance of elements whose subscript is $i$ on the elements whose subscript is $j$, and by the method of characteristic root, we can get weight vector :

$$
W_{i 1}{ }^{(j l)}, W_{i 2}{ }^{(j l)}, W_{i 3}{ }^{(j l)}, \ldots, W_{i n}{ }^{(j l)}
$$

For $\left(l=1,2, \ldots, n_{j}\right)$ repeat steps, get the matrix $W_{i j}$ :

$$
W_{i j}=\left[\begin{array}{cccc}
W_{i 1}^{(\mathrm{j} 1)} & W_{i 1}^{(\mathrm{j} 2)} & \mathrm{L} & W_{i 1}^{\left(\mathrm{jn}_{j}\right)} \\
W_{i 2}^{(\mathrm{j} 1)} & W_{i 2}^{(\mathrm{j} 2)} & \mathrm{L} & W_{i 2}^{\left(\mathrm{jn}_{j}\right)} \\
\mathrm{M} & & \mathrm{M} & \\
W_{i n_{i}}^{(\mathrm{j} 1)} & W_{i n_{i}}^{(\mathrm{j} 2)} & \cdots & W_{i n_{i}}^{\left(\mathrm{jn}_{j}\right)}
\end{array}\right]
$$

In the matrix $W_{i j}$, column vector $C_{i j}$ has elements $e_{i 1}, e_{i 2}, \ldots, e_{i n_{j}}$, row vector $C_{j}$ has elements $e_{j 1}, e_{j 2}, \ldots, e_{j n_{j}}$. Rank the effect $C_{i}$ on $C_{j}$, we find, there are $m$ super matrix under rule hierarchy $P_{s}$ :

$$
W=\left[\begin{array}{cccc}
W_{11} & W_{12} & \mathrm{~L} & W_{1 N} \\
W_{21} & W_{22} & \mathrm{~L} & W_{2 N} \\
\mathrm{M} & & & \mathrm{M} \\
W_{N 1} & W_{N 2} & \cdots & W_{N N}
\end{array}\right]
$$

In order to take impact of foreign elements to this criterion into account, we need perform normalization processing for super-matrix, and construct relative importance judgment matrix under rule hierarchy $P_{s}$. So, $N \times N$ step weighting matrix is:

$$
A=\left[\begin{array}{cccc}
a_{11} & a_{12} & \mathrm{~L} & a_{1 N} \\
a_{21} & a_{22} & \mathrm{~L} & a_{1 N} \\
\mathrm{M} & \multicolumn{2}{c}{\mathrm{M}} & \mathrm{M} \\
a_{N 1} & a_{N 1} \mathrm{~L} & a_{N N}
\end{array}\right]
$$

To reflect the dependencies between elements, implement stability operation on weighting super-matrix. Then Limit relative ranking vector is:

$$
W^{\infty}=\underline{\operatorname{Lim}}(1 / N) \sum_{K=1}^{N} W^{K}
$$

Thus, in our ANP separation model, we get the Partial weight vector:

$$
Q=\left[q_{11}, \ldots, q_{1 n_{1}}, q_{21}, \ldots, q_{2 n_{2}}, q_{N 1}, \ldots, q_{N n_{N}}\right]^{T}
$$

The final separation formula is expressed as:

$$
\begin{aligned}
& P=\sum_{N=1}^{n_{1}} q_{1_{N}} \cdot \text { Term }+\sum_{N=1}^{n_{2}} q_{2_{N}} \cdot \text { Abi. }+\sum_{N=1}^{n_{3}} q_{3_{N}} \cdot \text { Depart } . \\
& +\sum_{N=1}^{n_{4}} q_{4_{N}} \cdot \text { Level } \\
& +\sum_{N=1}^{n_{5}} q_{5_{N}} \cdot \text { Loy. }+\sum_{N=1}^{n_{6}} q_{6_{N}} \cdot \varepsilon
\end{aligned}
$$

\section{SEPARATION RATE INFLUENCE ON PRODUCTIVITY}

Human resource shortages caused by the departure of employees will directly impede their overall operational productivity. And indirect impediment to productivity include two aspects. One, with a large number of new employees to join, they aren't familiar with the corporate culture and tasks, so they need adaptation process for their own position. This process leads to low efficiency of their work, it will inevitably result in average operating decline, restricting the rapid development of enterprises. Two, company is always worried about being short-handed, marginal and poor employees are allowed to stay on in an attempt to lower the separation, therefore, very few employees are relieved or fired. This results in lower quality employees who often stay with the company for a full career. This part of the low quality employees do not match with their job, lead to the ineffective completion of work, and indirectly lower the enterprise productivity.

We introduce the variable "productivity impediment degree", to analyze the impact on human resources flow network model ${ }^{[\mathrm{v}]}$.

After analysis of some actual data, we can find the greater the degree of departure, the higher the directly productivity impediment degree can be. One people leave, will lead to further personnel fluctuation, cause a new round of departure, make human resources network model change. We choose one point as an example, to analyze the impact on the people around he/she when the point leave.

We define the concept of "departure infection degree ", said the individual's departure will make the people around he generate turnover intention in different degrees. According to the Field Theory of famous American psychologist Kurt Lewin ${ }^{[\mathrm{vi}]}$, individual performance and personal capacity, condition, there is a strong function similar to the relationship between physics field environment.

We only consider departure infection degree as the environmental factor, and we find that, the further the 
distance between two person $L$ is, the weaker the influence is. The relationship between distance and influence is showed ${ }^{[\text {vii] }}$ as the Fig.2. When one person leaves, separation seems to diffuse from employee to employee, it will increase the departure tend (Q).

$$
\begin{aligned}
& \Delta Q=k / L^{2} ; \\
& Q=Q_{0}+\Delta Q
\end{aligned}
$$

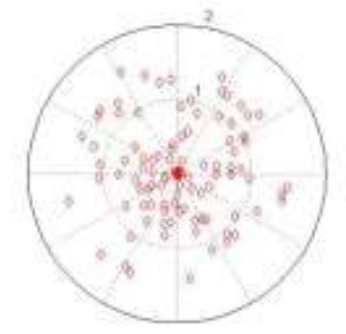

Figure 2. Distance-Influence Relationship

Indirectly productivity impediment factor is caused by mismatch between worker' $\mathrm{s}$ ability and position. Among them ${ }^{[\text {viii }]}$, part of the novices (transferred or employed ) may reach a basic level after receiving the appropriate training. But other part of them may still not be able to exert all their power (lack of productivity), because they are in the wrong position. The second situation even lead to the final transfer, which resulted in fluctuations in network systems. Compared with the direct factors, indirect factors' impact on fluctuations apparently is smaller.

We gives different weights for direct/indirect productivity impediment degree, said the triggered ability of network fluctuations.

\begin{tabular}{lll}
\hline & Direct & indirect \\
\hline Weights & 0.8 & 0.2 \\
\hline
\end{tabular}

TABLE II . WEIGHT ALLOCATION ON PRODUCTIVITY

Thus, the two Parameter above explained the triggered factors of HR network fluctuations. And we think the influence of these factors like water-flow ${ }^{[i x]}$, it can influent the lower level and the same level surrounding. Finally, we comprehensively analyzed ${ }^{\mathrm{l}}$ the impact of personnel-flow (different office) on the company, As shown in Fig.3:

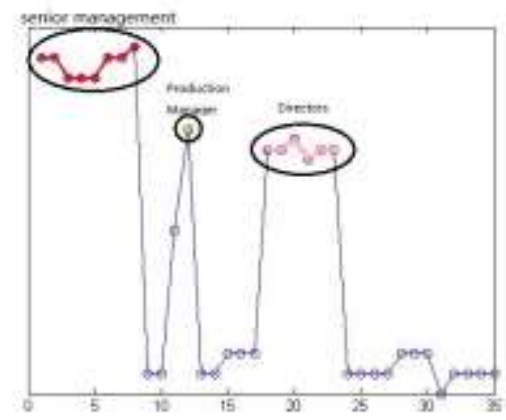

Figure 3.Separation trend in different employee level

It illustrates that, senior management, production manager and directors are important positions. If these people leave company, it will lead terrible influence to productivity.

\section{UNCONTROLLED SEPARATION RATE}

From the data ${ }^{[\mathrm{x}]}$ above, we find separation reach a high rate, the costs (training and recruitment) of two situations are almost the same! This arouses our reflection, what deep impact the growth of the separation rate on the company?

We must conclude the interact with each other. When the status share $\mathrm{e}^{[\mathrm{xi}]}$ decreases as high separation rate increases, "departure infection degree" should be taken into our network model. Because the negative effects cannot be ignored. So we have to improve the separation rate increasing model we have established, adding departure infection degree to it. In other words, separation rate increases to a certain high value, company can hardly control it. Then the separation rate will increase sharply, not stablely. So we improve our complex network model, and simulate the separation rate increasing tend once again. We find the trend curve has an "Anti-S" shape, the Fig. 4 following shows it.

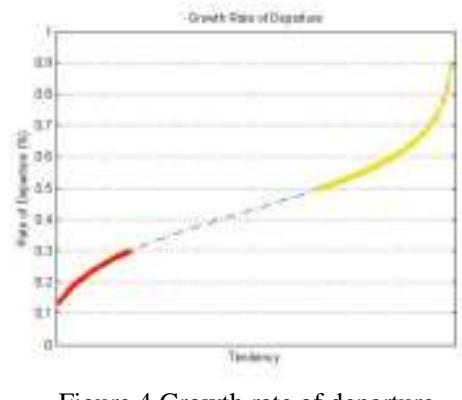

Figure 4.Growth rate of departure

We can clearly see, when the separation rate is lower $(<30 \%)$ as the red line, its growth increasing steadily. But if the company don't reduce the separation rate effectively, then the company will enter a dangerous period as the dotted line, maintaining a rapid growth. When the separation rate is too large $(>50 \%)$, it will increase sharply, and this situation will induce irreparable effects and even bankrupt!

\section{MULTI-LAYER NETWORKS}

To manage a company better, Human Captital Network is not the only factor. We hope to promote the company from many layers, such as information flow, trust, influence, and friendship. If company has these networks, then the HR can assess company from diversified aspects, which will detect inadequate tissue of organization timely. All in all, the multi-layer network is conducive to long-term development of the company.

But single network is dynamic, the relationship between two networks is dynamic, how could we to build multi-layer networks? How do we to get all these single networks together? We will explain our method from three steps as following. Find cluster members First, we divides the dynamic process into snapshots with same time interval. According to the conceptions such as similarity 
and centrality of vertices, every snapshot gets the corresponding clustering outcomes in a speed accelerated by an improved hierachical clustering algorithm.

- Find cluster members

First, we divides the dynamic process into snapshots with same time interval. According to the conceptions such as similarity and centrality of vertices, every snapshot gets the corresponding clustering outcomes in a speed accelerated by an improved hierachical clustering algorithm.

In details, we cluster the $i_{s t}$ snapshot, $C_{i}^{j}$ means the $j_{s t}$ community in the $i_{s t}$ snapshot, $n$ is the quantities of single-network. Then the result of cluster is:

$$
C_{i}=\left\{C_{i}^{1}, C_{i}^{2}, \mathrm{~L}, C_{i}^{n}\right\}
$$

We calculate Euclidean Distance between vertices(in single-network, each node is a vertex):

$$
d(i, j)=\sqrt{\sum_{k \neq i, j}\left(A_{i k}-A_{j k}\right)^{2}}
$$

$A$--the connection matrix of vertices;

$A_{i j}$--the weight on each link.

Then the weight of every node is :

$$
k_{i}=\sum_{j=1}^{m} A_{i j}
$$

The node with biggest weight is the center of the network, and the distance between two networks approximately equals to distance between their center :

$$
\operatorname{distc}\left(C^{i}, C^{j}\right) \approx d\left(f\left(C^{i}\right), f\left(C^{j}\right)\right)
$$

Cluster networks who has the shortest distance, until networks are combined to one community.

- Choose appropriate cluster member

According to Rand Index Function, we calculate the difference of cluster members:

$$
\operatorname{diff}\left(C_{i}\right)=\frac{\sum_{j=1, j \neq i}^{\text {numc }} R\left(C_{i}, C_{j}\right)}{\text { numc }-1}
$$

Then we calculate the average. If $\operatorname{diff}\left(C_{i}\right) \geq$ average, we will choose this member. If not, then we will abandon it.

\section{- Fusion}

With time goes by, networks change constantly, so we use the weighted Co-association matrix in term of time attenuation.

$$
\begin{aligned}
& C o(i, j)=\sum_{p=1, C_{p}=C}^{n u m c}\left(\frac{1}{n u m c^{\prime}} \times \frac{p}{n u m c} \times g(i, j, p)\right) \\
& \mathrm{g}(i, j, p)=\left\{\begin{array}{l}
1 \\
0
\end{array}\right.
\end{aligned}
$$

Among them

1: in $C_{p}, i, j$ belong to the same cluster;

0 : in $C_{p}, i, j$ don't belong to the same cluster;

numc -- the number of cluster members before choosing.

numc' --the number of cluster members after choosing.

Set Similarity threshold between two networks as $\mathrm{t}$, if $C_{o}(i, j) \phi t$,then combined them. The multi-layered networks we get is as below:
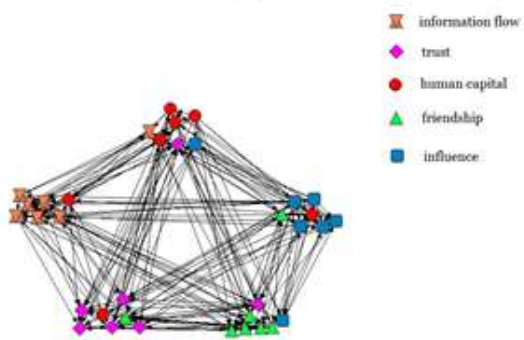

\section{SUMMARY}

In practice company management, simulation method should conclude local economic, region and other external factors, so as to express more effect.

\section{REFERENCES}

[1]Zhang Lanxia, Wangjun: Based on BP Network Human resouce management, 2012

[2].Robert S..How Risky is Your Company? Harvard Business Review,1999,(5-6):31-33.

[3]Science Reseach Management:Cooperation Network Analysis in Science Field,2011,Vol.32,No.12

[4]Lambert, Eric G ; Hogan, Nancy Lynne ; Barton, Shannon M (2001) . The impact of job satisfaction on turnover intent : a test of a structural measurement model using a national sample of workers. Social Science journal, 38,2,233-251

[5]Mansoureh Takaffoli,Farzad Sangi, Justin Fagnan, et al.Community Evolution Mining in Dynamic Social Networks[J],Procedia-Social and Behavioral Sciences, 2011,7(55):49-58.

[6]Kurt Lewin : Dynamic theory of personality (1935)

[6]Kurt Lewin : Dynamic theory of personality (1935)

[7] Yang Kai, Zhou Wei,Ma Li:Basis Application and the Realization of Simulation in MATLAB,(Southwest Jiaotong University Press,2012)

[8]Zhang Zhen, Liang Yongquan, Zhang Xinglin:Community Mining In Dynamic Co mplex Network : Selective Clustering Fusion Algorithm.Computer\&Digtal Engeering,Vol.41 No.3(2013)

[9]Bedeian A G, Ferris G R, Kacmar K M. Age, tenure, and job satisfaction : A tale of two perspectives [J]. Journal of Vocational Behaviour, 1992,40 : 33-48

[10]MehmedKantardzle((DataMiningConeePts,Models,Methods, andAlg orithms))IEEEpres

[11]Lihua Z, Ming Y. Analysis of Scientific Collaboration Network of China's Management Sciences Based on SNA--For the sample of management review(2004-2008) [J].Management Review. 2010,22(4): 39-46. 